Editorials

\title{
On Matrix Representation of Bernstein Polynomials for Triple Sequences
}

\author{
${ }^{1}$ Arulmani Indumathi, ${ }^{2}$ Ayhan Esi and ${ }^{1}$ Nagarajan Subramanian \\ ${ }^{1}$ Department of Mathematics, SASTRA Deemed to be University, Thanjavur-613 401, India \\ ${ }^{2}$ Department of Basic Engineering Sciences, Malatya Turgut Ozal University, 44040, Malatya, Turkey
}

Article history

Received: 29-06-2020

Revised: $15-08-2020$

Accepted: 21-08-2020

Corresponding Author:

Ayhan Esi

Department of Basic

Engineering Sciences, Malatya

Turgut Ozal University, 44040,

Malatya, Turkey

Email: aesi23@hotmail.com
Abstract: In this article, we give an overview of other commonly used basis for the triple sequence space of Bernstein polynomials, the Bernstein basis and put forward a matrix representation of Bernstein polynomials for the triple sequence space and its many useful properties.

Keywords: Matrix, Basis, Triple Sequences, Bernstein Polynomials, Power Basis

\section{Introduction}

Proof of the famous Weierstrass Approximation Theorem was found by (Bernstein, 1912). Polynomials, called Bernstein polynomials, are defined with the help of probability theory as follows:

Let $f:[0,1] \rightarrow R$. The Classical Bernstein polynomial $f$ is $B_{n}(f, x)=\sum_{k=0}^{n} f\left(\frac{k}{n}\right)\left(\begin{array}{l}n \\ k\end{array}\right) x^{k}(1-x)^{(n-k}, n=1,2, \ldots$. Later, Bernstein polynomials, bringing them into an intensive field of research were found to have many remarkable features.

In this article, we have studied polynomials that are simply defined, incredibly useful mathematical tools that can be calculated quickly in computer systems and representing a wide variety of functions. Consider that:

$$
p(x)=a_{0}+a_{1} x+a_{2} x^{2}+\cdots+a_{n} x^{n}
$$

as a linear combination of certain basic polynomial represents a polynomial $\left\{\left(1, x, x^{2}, x^{3}, \cdots x^{n}\right)\right\}$. Generally, a polynomial function with a degree less than or equal to $n$, can be expressed as:

i. A set of polynomials equal to or less than $n$ forms a vector space: Polynomials can be added together, multiplied by a scalar and all vector space properties apply

ii. (ii) The set of functions $\left\{\left(1, x, x^{2}, x^{3}, \cdots x^{n}\right)\right\}$ forms a basis for this vector space - that is, the polynomial of any degree less than or equal to $n$ is uniquely a linear combination
Often called the power basis, this basis is just one of the infinitely many bases for the triple sequence space of Bernstein polynomials. Bernstein polynomials play an important role in other areas of mathematics and approximation theory. They also play an important role in physics. So now we give the definition of these polynomials and their important properties:

1. They are non-negative throughout the interval $[a, b]$

2. They are symmetric.

We can write $B_{r s t}(f, x)=B_{r-m, s-n, t-k}(a+b-a(f, x))$

3. Each continuous polynomial has only one maximum in the interval $[a, b]$ at $x=a+\frac{(b-a) m}{r}+\frac{(b-a) n}{s}+$ $\frac{(b-a) k}{t}$, according to the interval value theorem

4. The set of degrees $(r, s, t)$ of these polynomials form part of the unity as $\sum_{m=0}^{r} \sum_{n=0}^{s} \sum_{k=0}^{t} B_{r s t}(f, x)=1$

5. Always a Bernstein polynomial can be expressed as a linear combination of polynomials of higher degree as follows:

$$
\begin{aligned}
& B_{(r-1)(s-1)(t-1)}(f, x)=\left(\frac{r-m}{r}\right)\left(\frac{s-n}{s}\right)\left(\frac{t-k}{t}\right) B_{r s t}(f, x) \\
& +\left(\frac{m+1}{r}\right)\left(\frac{n+1}{s}\right)\left(\frac{k+1}{t}\right) B_{r s t}(f, x) .
\end{aligned}
$$

There are $(r s t)^{\text {th }}$ degree Bernstein polynomials. For mathematical convention, we usually set $B_{r s t}=0$ if $m, n$, $k<0$ or $m>r, n>s, k>t$. Moreover, the Bernstein 
polynomials can be defined in term of forward differences as follows:

$$
\begin{aligned}
& B_{r s t}(f, x) \\
& =\sum_{m=0}^{r} \sum_{n=0}^{s} \sum_{k=0}^{t} \Delta^{m n k} f(0)\left(\begin{array}{l}
r \\
m
\end{array}\right)\left(\begin{array}{l}
s \\
n
\end{array}\right)\left(\begin{array}{l}
t \\
k
\end{array}\right) x^{m+n+k}(1-x)^{(m-r)+(n-s)+(k-t)} .
\end{aligned}
$$

Let $K$ be a subset of the set of positive integers $\mathbb{N} \times \mathbb{N} \times \mathbb{N}$ and let us denote the set $\{(m, n, k) \in K: m \leq u$, $n \leq v, k \leq w\}$ by $K_{u v w}$. Then the natural density of $K$ is given by $\delta(K)=\lim _{u, v, w \rightarrow \infty} \frac{\left|K_{u v w}\right|}{u v w}$, where $\left|K_{u v w}\right|$ denotes the number of elements in $K_{u v w}$. Clearly, a finite subset has natural density zero and we have $\delta\left(K^{c}\right)=1-\delta(K)$ where $K^{c}=\mathbb{N} \backslash K$ is the complement of $K$. If $K_{1} \subseteq K_{2}$, then $\delta\left(K_{1}\right) \leq \delta\left(K_{2}\right)$.

The Bernstein operator of order rst is given by:

$$
\begin{aligned}
& B_{r s t}(f, x) \\
& =\sum_{m=0}^{r} \sum_{n=0}^{s} \sum_{k=0}^{t} f\left(\frac{m n k}{r s t}\right)\left(\begin{array}{c}
r \\
m
\end{array}\right)\left(\begin{array}{l}
s \\
n
\end{array}\right)\left(\begin{array}{l}
t \\
k
\end{array}\right) x^{m+n+k}(1-x)^{(m-r)+(n-s)+(k-t)}
\end{aligned}
$$

where, $f$ is a continuous (real or complex valued) function defined on $[0,1]$ and:

$$
\left(\begin{array}{c}
r \\
m
\end{array}\right)\left(\begin{array}{l}
s \\
n
\end{array}\right)\left(\begin{array}{l}
t \\
k
\end{array}\right)=\frac{r !}{m !(r-m) !} \frac{s !}{n !(s-n) !} \frac{r !}{t !(t-k) !} .
$$

Throughout the paper, $\mathbb{R}$ denotes the threedimensional real space with the $(X, d)$ metric. Consider a triple sequence $x=\left(x_{m n k}\right)$ such that $x_{m n k} \in \mathbb{R}, m, n, k \in \mathbb{N}$.

Let $f$ be a continuous function defined on the interval $[0,1]$. A triple sequence of Bernstein polynomials $\left(B_{r s t}(f, x)\right)$ is said to be statistically convergent to $0 \in \mathbb{R}$, denoted by $s t-\lim x=0$, provided that the set:

$$
K_{\varepsilon}:=\left\{(m, n, k) \in \mathbb{N}^{3}:\left|B_{m n k}(f, x)-f(x)\right| \geq \varepsilon\right\}
$$

has natural density zero for any $\varepsilon>0$. In this case, 0 is called the statistical limit of the triple sequence of Bernstein polynomials. i.e., $\delta\left(K_{\varepsilon}\right)=0$. That is,

$$
\lim _{r, s, t \rightarrow \infty} \frac{1}{r s t}\left|\left\{m \leq r, n \leq s, k \leq t:\left|B_{m n k}(f, x)-f(x)\right| \geq \varepsilon\right\}\right|=0 .
$$

In this case, we write $\delta$ - $\lim B_{m n k}(f, x)=(f, x)$ or $B_{m n k}(f$, $x) \rightarrow s_{B} f(x)$.

Let $f$ be a continuous function defined on the interval $[0,1]$. A triple sequence of Bernstein polynomials
$\left(B_{r s t}(f, x)\right)$ is said to be statistically analytic if there is a positive number $M$ such that:

$$
\delta\left(\left\{(m, n, k) \in \mathbb{N}^{3}:\left|B_{m n k}(f, x)-f(x)\right|^{1 / m+n+k} \geq M\right\}\right)=0
$$

That is:

$$
\lim _{r, s, t \rightarrow \infty} \frac{1}{r s t}\left|\left\{(m, n, k) \leq(r, s, t):\left|B_{m n k}(f, x)-f(x)\right|^{1 / m+n+k} \geq M\right\}\right|=0 .
$$

A triple sequence (real or complex) can be defined as a function $x: \mathbb{N} \times \mathbb{N} \times \mathbb{N} \rightarrow \mathbb{R}(\mathbb{C})$, where $\mathbb{N}, \mathbb{R}$ and $\mathbb{C}$ represent the set of natural numbers, real numbers and complex numbers respectively. Different types of the triple sequence concept were introduced and first explored by (Sahiner et al., 2007; Sahiner and Tripathy, 2008; Esi, 2014; Esi and Catalbas, 2014; Esi and Savas, 2015; Esi and Sapszoglu, 2012; Esi and Subramanian, 2018b; Esi et al., 2017a; 2017b; Dutta et al., 2013; Subramanian and Esi, 2015; 2017a; 2017b; Esi and Subramanian, 2018a; Subramanian and Esi, 2018; Velmurugan and Subramanian, 2018; Hazarika et al., 2018; Subramanian et al., 2019; 2018; Debnath et al., 2015; Aiyub et al., 2017; Sharma and Esi, 2013), many others.

\section{A Basis of the Bernstein Polynomials of Triple Sequence Spaces}

Why is the basically triple sequence of Bernstein polynomials, Bernstein polynomials of order $n$ a basis for the triple sequence of polynomials of order $n$ ! equal to or lower?

(i) It covers the triple sequence space of polynomials any polynomial of order less than or equal to $n$ can be written as a linear combination of Bernstein polynomials

This easily covers triple sequence space of polynomials and the power basis of any member of the Bernstein polynomials in terms of power can be written as a linear combination of notice

(ii) They are linearly independent-that is, there exist constants $c_{0}, c_{1}, c_{2} \cdots c_{n}$ so that the identity:

$$
0=C_{0} B_{000, r s t}(x)+C_{1} B_{111, r s t}(x)+\cdots+C_{n} B_{m n k, r s t}(x)
$$

holds for all $t$, then all $m n k, c_{i}^{\prime} s$ must be zero.

If this is true, we could write: 


$$
\begin{aligned}
& 0=C_{0} B_{000, r s t}(x)+C_{1} B_{11, r s t}(x)+\cdots+C_{n} B_{m n k, r s t}(x) . \\
& =C_{0} \sum_{m=0}^{r} \sum_{n=0}^{s} \sum_{k=0}^{t}(-1)^{r+s+t}\left(\begin{array}{l}
r \\
m
\end{array}\right)\left(\begin{array}{l}
m \\
0
\end{array}\right)\left(\begin{array}{l}
s \\
n
\end{array}\right)\left(\begin{array}{l}
n \\
0
\end{array}\right)\left(\begin{array}{l}
t \\
k
\end{array}\right)\left(\begin{array}{l}
k \\
0
\end{array}\right) x^{m+n+k}+ \\
& C_{1} \sum_{m=1}^{r} \sum_{n=1}^{s} \sum_{k=1}^{t}(-1)^{(r-1)+(s-1)+(t-1)}\left(\begin{array}{l}
r \\
m
\end{array}\right)\left(\begin{array}{l}
m \\
1
\end{array}\right)\left(\begin{array}{l}
s \\
n
\end{array}\right)\left(\begin{array}{l}
n \\
1
\end{array}\right)\left(\begin{array}{l}
t \\
k
\end{array}\right)\left(\begin{array}{l}
k \\
1
\end{array}\right) x^{m+n+k}+\cdots+ \\
& C_{n} \sum_{m=r}^{r} \sum_{n=s}^{s} \sum_{k=t}^{t}(-1)^{(m-r)+(n-s)+(k-t)}\left(\begin{array}{l}
r \\
m
\end{array}\right)\left(\begin{array}{l}
m \\
r
\end{array}\right)\left(\begin{array}{l}
s \\
n
\end{array}\right)\left(\begin{array}{l}
n \\
s
\end{array}\right)\left(\begin{array}{l}
t \\
k
\end{array}\right)\left(\begin{array}{l}
k \\
t
\end{array}\right) x^{m+n+k} . \\
& =C_{0}+\left[C_{1} \sum_{m=0}^{1} \sum_{n=0}^{1} \sum_{k=0}^{1}\left(\begin{array}{l}
r \\
1
\end{array}\right)\left(\begin{array}{l}
1 \\
1
\end{array}\right)\left(\begin{array}{l}
s \\
1
\end{array}\right)\left(\begin{array}{l}
1 \\
1
\end{array}\right)\left(\begin{array}{l}
t \\
1 \\
t
\end{array}\right)\left(\begin{array}{l}
1 \\
1
\end{array}\right) x^{1}\right]+\cdots+ \\
& {\left[C_{n} \sum_{m=0}^{r} \sum_{n=0}^{s} \sum_{k=0}^{t}\left(\begin{array}{l}
r \\
r
\end{array}\right)\left(\begin{array}{l}
r \\
r
\end{array}\right)\left(\begin{array}{l}
s \\
s
\end{array}\right)\left(\begin{array}{l}
s \\
s
\end{array}\right)\left(\begin{array}{l}
t \\
t
\end{array}\right)\left(\begin{array}{l}
t \\
t
\end{array}\right) x^{r+s+t}\right] .}
\end{aligned}
$$

Since the power basis is a linearly independent set, we must have that:

$C_{0}=0$

$$
\begin{aligned}
& C_{1} \sum_{m=0}^{1} \sum_{n=0}^{1} \sum_{k=0}^{1}\left(\begin{array}{l}
r \\
1
\end{array}\right)\left(\begin{array}{l}
1 \\
1
\end{array}\right)\left(\begin{array}{l}
s \\
1
\end{array}\right)\left(\begin{array}{l}
1 \\
1
\end{array}\right)\left(\begin{array}{l}
t \\
1
\end{array}\right)\left(\begin{array}{l}
1 \\
1
\end{array}\right)=0 \\
& C_{n} \sum_{m=0}^{r} \sum_{n=0}^{s} \sum_{k=0}^{t}\left(\begin{array}{l}
r \\
r
\end{array}\right)\left(\begin{array}{l}
r \\
r
\end{array}\right)\left(\begin{array}{l}
s \\
s
\end{array}\right)\left(\begin{array}{l}
s \\
s
\end{array}\right)\left(\begin{array}{l}
t \\
t
\end{array}\right)\left(\begin{array}{l}
t \\
t
\end{array}\right)=0
\end{aligned}
$$

which implies that $C_{0}=C_{1}=C_{2}=\cdots=C_{n}=0 .\left(C_{0}\right.$ is clearly zero, substituting this in the second equation gives $C_{1}=0$, substituting these two into the third equation gives ...).
The Bernstein polynomials of degree (rst) can be defined by blending two Bernstein polynomials of degree $n=1$. So, the $(m n k)^{\text {th }}(r s t)^{\text {th }}$ degree Bernstein polynomials can be written as:

$$
\begin{aligned}
& B_{r s t}(x)=(1-x) B_{m n k, r-1, s-1, t-1}(x) \\
& +x B_{(m-1, n-1, k-1),(r-1, s-1, t-1)}(x) .
\end{aligned}
$$

for $0 \leq m \leq r, 0 \leq n \leq s, 0 \leq k \leq t$ and the derivative of the $(r s t)^{\text {th }}$ degree Bernstein polynomials are also polynomials of degree $(r-1, s-1, t-1)$ and they are defined as follows:

$$
\begin{aligned}
& \frac{d}{d x} B_{m n k, r s t}(x) \\
& =r s t\left[B_{(m-1, n-1, k-1),(r-1, s-1, k-1)}(x)-B_{m m k,(r-1, s-1, k-1)}(x)\right]
\end{aligned}
$$

\section{Bernstein Polynomials for Triple Sequence Spaces}

The Bernstein polynomials of degree $r s t$ are defined by:

$$
\begin{aligned}
& B_{m n k, r s t}(f, x) \\
& =\sum_{m=0}^{r} \sum_{n=0}^{0} \sum_{k=0}^{t} f\left(\frac{m n k}{r s t}\right)\left(\begin{array}{l}
r \\
m
\end{array}\right)\left(\begin{array}{l}
s \\
n
\end{array}\right)\left(\begin{array}{l}
t \\
k
\end{array}\right) x^{m+n+k}(1-x)^{(r-m)+(s-n)+(t-k)}
\end{aligned}
$$

for $m, n, k=0,1,2, \cdots, r, s, t$.
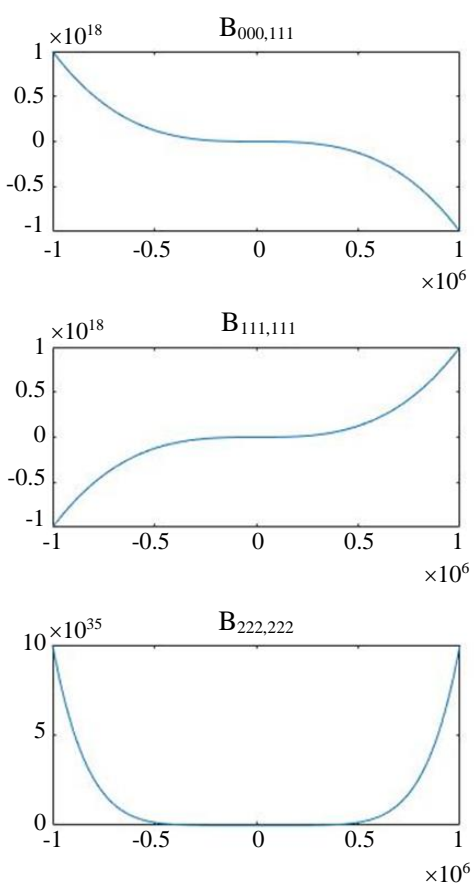
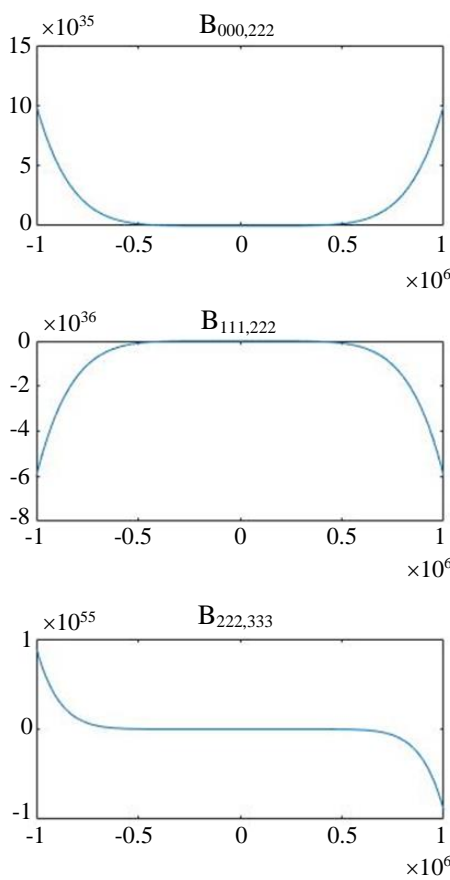
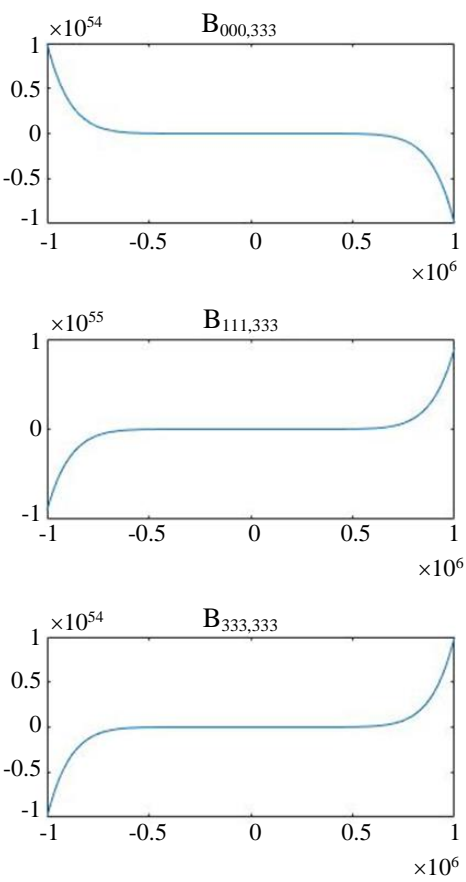

Fig. 1: The triple sequence of Bernstein Polynomials of degrees 1, 2 and 3 
There are $(r+1)(s+1)(t+1),(r s t)^{\text {th }}$ degree Bernstein polynomials for mathematical compatibility we usually set $B_{m n k, r s t}=0$ for $m, n, k<0$ or $m>r, n>s, k>t$. It is quite easy to write the coefficients of these polynomials $\left(\begin{array}{c}r \\ m\end{array}\right)\left(\begin{array}{l}s \\ n\end{array}\right)\left(\begin{array}{l}t \\ k\end{array}\right)$ from Pascal's triangle, the exponents in the $x$ term increase as $m, n, k$ increases and the exponents on the $(1-x)$ term decrease by one as $m, n, k$ increases. In simple cases, we get (Fig. 1):

- The triple sequences of Bernstein polynomials of degree 1 are:

$$
B_{000,111}(x)=(1-x)^{3}, B_{111,111}(x)=x^{3} \text {. }
$$

- The triple sequences of Bernstein polynomials of degree 2 are:

$$
B_{000,222}(x)=(1-x)^{6}, B_{111,222}(x)=6 x^{3}(1-x)^{3}, B_{222,222}(x)=x^{6} .
$$

- The triple sequence of Bernstein polynomials of degree 3 are:

$$
\begin{aligned}
& B_{000,333}(x)=(1-x)^{9}, B_{111,333}(x)=9 x^{3}(1-x)^{6}, \\
& B_{222,333}(x)=9 x^{6}(1-x)^{3}, B_{333,333}(x)=x^{9}
\end{aligned}
$$

\section{Converting the Bernstein Basis to the Power Basis for Triple Sequence Spaces}

Since the power basis $\left\{1, x, x^{2}, x^{3} \cdots, x^{n}\right\}$ forms a basis for the triple sequence of Bernstein polynomials of degree less than or equal to $n$, any Bernstein polynomial of degree $n$ can be written in term of the power basis. This direct Bernstein polynomial can be calculated using the following definition of the binomial theorem:

$$
\begin{aligned}
& B_{m n k, r s t}(f, x)=\sum_{m=0}^{r} \sum_{n=0}^{s} \sum_{k=0}^{t} \\
& \left(\begin{array}{l}
r \\
m
\end{array}\right)\left(\begin{array}{l}
s \\
n
\end{array}\right)\left(\begin{array}{l}
t \\
k
\end{array}\right) x^{m+n+k}(1-x)^{(r-m)+(s-n)+(t-k)} \\
& =\left(\begin{array}{l}
r \\
u
\end{array}\right)\left(\begin{array}{l}
s \\
v
\end{array}\right)\left(\begin{array}{l}
t \\
w
\end{array}\right) x^{u+v+w} \sum_{m=0}^{r-u} \sum_{n=0}^{s-v} \sum_{k=0}^{t-w}(-1)^{m+n+k}\left(\begin{array}{c}
r-u \\
m
\end{array}\right)\left(\begin{array}{c}
s-v \\
n
\end{array}\right)\left(\begin{array}{c}
t-w \\
k
\end{array}\right) x^{m+n+k} \\
& =\sum_{m=0}^{r-u} \sum_{n=0}^{s-v} \sum_{k=0}^{t-w}(-1)^{m+n+k} \\
& \left(\begin{array}{l}
r \\
u
\end{array}\right)\left(\begin{array}{c}
s \\
v
\end{array}\right)\left(\begin{array}{c}
t \\
w
\end{array}\right)\left(\begin{array}{c}
r-u \\
m
\end{array}\right)\left(\begin{array}{c}
s-v \\
n
\end{array}\right)\left(\begin{array}{c}
t-w \\
k
\end{array}\right) x^{(m+u)+(n+v)+(k+w)} \\
& =\sum_{m=0}^{r} \sum_{n=0}^{s} \sum_{k=0}^{t}(-1)^{(m-u)+(n-v)+(k-w)} \\
& \left(\begin{array}{l}
r \\
u
\end{array}\right)\left(\begin{array}{c}
s \\
v
\end{array}\right)\left(\begin{array}{c}
t \\
w
\end{array}\right)\left(\begin{array}{c}
r-u \\
m-u
\end{array}\right)\left(\begin{array}{c}
s-v \\
n-v
\end{array}\right)\left(\begin{array}{c}
t-w \\
k-w
\end{array}\right) x^{m+n+k} \\
& =\sum_{m=0}^{r} \sum_{n=0}^{s} \sum_{k=0}^{t}(-1)^{(m-u)+(n-v)+(k-w)} \\
& \left(\begin{array}{c}
r \\
m
\end{array}\right)\left(\begin{array}{l}
s \\
n
\end{array}\right)\left(\begin{array}{l}
t \\
k
\end{array}\right)\left(\begin{array}{l}
m \\
u
\end{array}\right)\left(\begin{array}{c}
n \\
v
\end{array}\right)\left(\begin{array}{c}
k \\
w
\end{array}\right) x^{m+n+k} .
\end{aligned}
$$

We have used the binomial theorem to expand $(1-x)^{(r-}$ $u)+(s-v)+(t-w)$

To prove that each power basis element can be expressed as a linear combination of Bernstein polynomials, we use the degree elevation formulas and induction to calculate the following:

$$
\begin{aligned}
& x^{u+v+w}=x\left(x^{(u-1)}+x^{(v-1)}+x^{(w-1)}\right) \\
& =x \sum_{m=0}^{r} \sum_{n=0}^{s} \sum_{k=0}^{t} \frac{\left(\begin{array}{c}
m \\
u-1
\end{array}\right)\left(\begin{array}{c}
n \\
v-1
\end{array}\right)\left(\begin{array}{c}
k \\
w-1
\end{array}\right)}{\left(\begin{array}{c}
r \\
u-1
\end{array}\right)\left(\begin{array}{c}
s \\
v-1
\end{array}\right)\left(\begin{array}{c}
t \\
w-1
\end{array}\right)} B_{m n k,(r-1)(s-1)(t-1)}(x) \\
& =x \sum_{m=u}^{r} \sum_{n=v}^{s} \sum_{k=w}^{t} \frac{\left(\begin{array}{c}
m-1 \\
u-1
\end{array}\right)\left(\begin{array}{c}
n-1 \\
v-1
\end{array}\right)\left(\begin{array}{c}
k-1 \\
w-1
\end{array}\right)}{\left(\begin{array}{c}
r-1 \\
u-1
\end{array}\right)\left(\begin{array}{c}
s-1 \\
v-1
\end{array}\right)\left(\begin{array}{c}
t-1 \\
w-1
\end{array}\right)} B_{(m-1)(n-1)(k-1),(r-1)(s-1)(t-1)}(x) \\
& =\sum_{m=u-1}^{r-1} \sum_{n=v-1}^{s-1} \sum_{k=w-1}^{t-1} \frac{\left(\begin{array}{l}
m \\
u-1
\end{array}\right)\left(\begin{array}{c}
n \\
v-1
\end{array}\right)\left(\begin{array}{c}
r \\
w-1 \\
u-1
\end{array}\right)\left(\begin{array}{c}
s \\
v-1
\end{array}\right)\left(\begin{array}{c}
t \\
w-1
\end{array}\right)}{(m n k)} \frac{(r s t)}{\left(\begin{array}{l}
w \\
w n k, r s t
\end{array}\right)}(x) \\
& =\sum_{m=u-1}^{r-1} \sum_{n=v-1}^{s-1} \sum_{k=w-1}^{t-1} \frac{\left(\begin{array}{l}
m \\
u
\end{array}\right)\left(\begin{array}{l}
n \\
v
\end{array}\right)\left(\begin{array}{l}
k \\
w
\end{array}\right)}{\left(\begin{array}{l}
r \\
u
\end{array}\right)\left(\begin{array}{l}
s \\
v
\end{array}\right)\left(\begin{array}{l}
t \\
w
\end{array}\right)} B_{m n k, r s t}(x),
\end{aligned}
$$

where the induction hypothesis was used.

\section{A Matrix Representation of Bernstein Polynomials for Triple Sequence Spaces}

For example, until the $1990 \mathrm{~s}$ as a systematic review of Bernstein polynomials theory (Lorentz, 1986; Videnskii and Polynomials, 1990) are also presented. New articles are constantly coming out (Esi and Catalbas, 2014) and new applications and generalizations are discovered (Li et al., 1997; Petrone, 1999). A generalization of Bernstein polynomials containing q-integers was proposed by (Lupas, 1987). However, the q-analogue of the Bernstein operator as handled by Lupa-s gives rational functions rather than polynomials.

In many applications, it is helpful to Bernstein polynomials of a matrix formulation. When looking at only a linear combination in terms of point products, they are simple to develop.

Given a polynomial that can be expressed as a linear combination of Bernstein's elementary functions:

$$
B(t)=C_{0} B_{000, r s t}(x)+C_{1} B_{111, r s t}(x)+\cdots+C_{n} B_{m n k, r s t}(x) .
$$

It's easy to express this as the dot product of two vectors: 


$$
B(t)=\left[B_{000, r s t}(x) B_{111, r s t}(x) \cdots B_{m n k, r s t}(x)\right]\left[\begin{array}{l}
C_{0} \\
C_{1} \\
\vdots \\
C_{n}
\end{array}\right]
$$

We can convert this to:

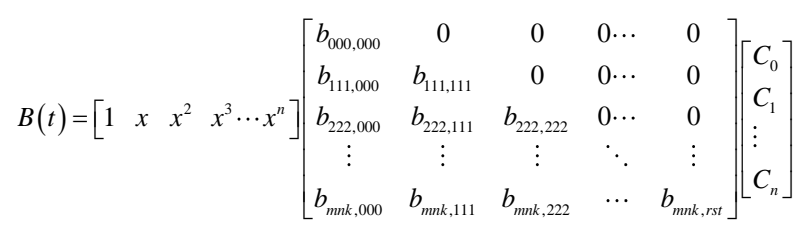

where the $b_{i j k}$ are the coefficients of the power base used to determine the respective Bernstein polynomials. Notice that in such a case the matrix is the lower triangular matrix.

In the quintic case $(n=5)$, the matrix representation is:

$$
B(t)=\left[\begin{array}{llllll}
1 & x & x^{2} & x^{3} & x^{4} & x^{5}
\end{array}\right]\left[\begin{array}{cccccc}
1 & 0 & 0 & 0 & 0 & 0 \\
-5 & 5 & 0 & 0 & 0 & 0 \\
5 & -6 & 5 & 0 & 0 & 0 \\
-1 & 5 & -5 & 1 & 0 & 0 \\
4 & -5 & 4 & -4 & 5 & 0 \\
-3 & 6 & -4 & 4 & -5 & 3
\end{array}\right]\left[\begin{array}{l}
C_{0} \\
C_{1} \\
C_{2} \\
C_{3} \\
C_{4} \\
C_{5}
\end{array}\right]
$$

and in the sextic case $(n=6)$, the matrix representation is:

$$
B(t)=\left[\begin{array}{llllll}
x^{6} & x^{6} & x^{6} & x^{6} & x^{6} & x^{6}
\end{array}\right]\left[\begin{array}{ccccccc}
1 & 0 & 0 & 0 & 0 & 0 & 0 \\
-5 & 5 & 0 & 0 & 0 & 0 & 0 \\
5 & -6 & 5 & 0 & 0 & 0 & 0 \\
-1 & 5 & -5 & 1 & 0 & 0 & 0 \\
4 & -5 & 4 & -4 & 5 & 0 & 0 \\
-3 & 6 & -4 & 4 & -5 & 3 & 0 \\
7 & -8 & 4 & -3 & 5 & -3 & 7
\end{array}\right]\left[\begin{array}{c}
C_{0} \\
C_{1} \\
C_{2} \\
C_{3} \\
C_{4} \\
C_{5} \\
C_{6}
\end{array}\right]
$$

\section{Acknowledgement}

As authors, we thank the referee for his/her contribution to our article.

\section{Author's Contributions}

All authors contributed equally to the preparation of the article.

\section{Competing Interests}

There are no conflict of interests to disclose for any of the authors of this study.

\section{References}

Aiyub, M., Esi, A., \& Subramanian, N. (2017). The triple entire difference ideal of fuzzy real numbers over fuzzy p-metric spaces defined by Musielak Orlicz function. Journal of Intelligent \& Fuzzy Systems, 33(3), 1505-1512.

Bernstein, S. (1912). Démo istration du th'eorème de Weierstrass fondée sur le calcul des probabilités. Сообщенія Харьковскаго математическаго общества, 13(1), 1-2.

Dutta, A. J., Esi, A. Y. H. A. N., \& Tripathy, B. C. (2013). Statistically convergent triple sequence spaces defined by Orlicz function. Journal of mathematical analysis, 4(2), 16-22.

Debnath, S., Sarma, B., \& Das, B. (2015). Some generalized triple sequence spaces of real numbers. Journal of Nonlinear Analysis and Optimization: Theory \& Applications, 6(1), 71-79.

Esi, A. (2014). On some triple almost lacunary sequence spaces defined by Orlicz functions. Research \& Reviews: Discrete Mathematical Structures, 1(2), 16-25.

Esi, A., \& Catalbas, M. N. (2014). Almost convergence of triple sequences. Global journal of mathematical analysis, 2(1), 6-10.

Esi, A., \& Sapszoglu, A. (2012). On some lacunary $\sigma$ strong Zweier convergent sequence spaces. Romai J, $8(2), 61-70$.

Esi, A., \& Savas, E. (2015). On lacunary statistically convergent triple sequences in probabilistic normed space. Applied mathematics \& information sciences, 9(5), 2529.

Esi, A., \& Subramanian, N. (2018a). Generalized Rough Cesaro and Lacunary Statistical Triple Difference Sequence Spaces in Probability of Fractional Order Defined by Musielak-Orlicz Function. International Journal of Analysis and Applications, 16(1), 16-24.

Esi, A., \& Subramanian, N. (2018b). On triple sequence spaces of Bernstein operator of [[chi]. sup. 3] of rough [lambda]-statistical convergence in probability of random variables defined by Musielak-Orlicz function. International Journal of Open Problems in Computer Science and Mathematics, 11(2), 62-71.

Esi, A., Araci, S., \& Esi, A. (2017a). $\lambda$-Statistical Convergence of Bernstein polynomial sequences. Advances and applications in mathematical sciences, 16(3), 113-119.

Esi, A., Subramanian, N., \& Esi, A. (2017b). Triple rough statistical convergence of sequence of Bernstein operators. Int. J. Adv. Appl. Sci, 4(2), 28-34. 
Hazarika, B., Subramanian, N., \& Esi, A. (2018). On rough weighted ideal convergence of triple sequence of Bernstein polynomials. In Proceedings of the Jangjeon Mathematical Society (Vol. 21, No. 3, pp. 497-506).

Li, X., Mikusiński, P., Sherwood, H., \& Taylor, M. D. (1997). On approximation of copulas. In Distributions with given marginals and moment problems (pp. 107-116). Springer, Dordrecht.

Lorentz, G. G. (1986). Bernstein Polynomials, Chelsea Publ. Co., New York.

Lupas, A. (1987). A q-analogue of the Bernstein operator, University of Cluj-Napoca Seminar on Numerical and Statistical Calculus. Preprint, 9, 85-92.

Petrone, S. (1999). Random bernstein polynomials. Scandinavian Journal of Statistics, 26(3), 373-393.

Sahiner, A., \& Tripathy, B. C. (2008). Some I related properties of triple sequences. Selcuk J. Appl. Math, 9(2), 9-18.

Sahiner, A., Gürdal, M., \& Düden, F. K. (2007). Triple sequences and their statistical convergence.

Sharma, S. K., \& Esi, A. (2013). Some I-convergent sequence spaces defined by using sequence of moduli and n-normed space. Journal of the Egyptian Mathematical Society, 21(2), 103-107.

Subramanian, N., \& Esi, A. (2015). The generalized tripled difference of $\chi 3$ sequence spaces. Global Journal of mathematical analysis, 3(2), 54-60.
Subramanian, N., \& Esi, A. (2017a). Rough variables of convergence. Vasile Alecsandri University of Bacau Faculty of Sciences, Scientific studies and Research series Mathematics and informatics, 27(2), 65-72.

Subramanian, N., \& Esi, A. (2017b). Wijsman rough convergence of triple sequences. Matematychni studii, 48(2), 171-179.

Subramanian, N., \& Esi, A. (2018). On triple sequence space of Bernstein operator of $\chi^{3}$ of rough $\lambda-$ statistical convergence in probability defined by Musielak-Orlicz function of $\mathrm{p}-$ metric. Electronic Journal of Mathematical Analysis and Applications, 6(1), 198-203.

Subramanian, N., Esi, A., \& Khan, V. A. (2018). The rough intuitionistic fuzzy Zweier lacunary ideal convergence of triple sequence spaces. Journal of Mathematics and Statistics, 10, 72-78.

Subramanian, N., Esi, A., \& Ozdemir, M. K. (2019). Rough statistical convergence on triple sequence of the Bernstein operator of random variables in probability. Songklanakarin Journal of Science \& Technology, 41(3).

Velmurugan, S., \& Subramanian, N. (2018). Bernstein operator of rough $\lambda$ - statistically and $\rho$ Cauchy sequences convergence on triple sequence spaces. Journal of Indian Mathematical Society, 85(1-2), 257-265.

Videnskii, V. S., \& Polynomials, B. (1990). Leningrad State Pedagocical University. Leningrad, Russia. 